\title{
Spontaneous Drainage of Lower Lip Abscess by Insect Sting in the Prediabetic Male: A Case Report
}

\author{
Meircurius Dwi Condro Surboyo, DDS, MDS, Bagus Soebadi, DDS, MHPED, \\ Hening Tuti Hendarti, DDS, MS and Desiana Radithia, DDS, PhD \\ Department of Oral Medicine, Faculty of Dental Medicine, Universitas Airlangga, Surabaya, Indonesia
}

\begin{abstract}
Most insects have a stinging apparatus at the tail end of their abdominal segment and can deliver venom. The venom can usually result in pain, erythema, abscess, or allergic reaction in human tissues such as the skin and even the lips. The presentation of lip abscess, especially in the vermilion, is rare and needs to be appropriately managed.

A 39-year-old prediabetic male presented with a swollen right lower lip stung by an insect six days before the consult. The swelling started as a vesicle which became bigger, then burst, exuding a yellowish fluid and blood. The swollen area became more significant, and pain was felt. The clinical presentation was that of a lower lip abscess. Adequate antibiotics were prescribed along with a corticosteroid and a topical antiseptic. The treatment was essential to prevent the dissemination of infection and the likelihood of tissue necrosis.

This case report is presented as a rare clinical case of a lower lip abscess due to an insect sting with spontaneous drainage after treatment in a prediabetic male. The dentist and clinician needed to identify this condition and to manage the case properly.
\end{abstract}

Key Words: insect sting, lip abscess, spontaneous drainage

\section{INTRODUCTION}

Lips reveal a heterogeneous group of lesions ranging from developmental, inflammatory, or neoplastic conditions. The most common cause for lip swelling is trauma, infection, and angioedema, causing transient swelling. ${ }^{1}$

Most insects may be dangerous to people because of their venom. The venom has substances and enzymes such as hyaluronidase, phospholipase A2, and Melittin, which can destroy the cell membrane, degranulate mast cells, and release immunoglobulin $\mathrm{E}(\mathrm{IgE})$, resulting in inflammation and allergic reactions. ${ }^{2}$ The venom enters the tissue through sting or bite. Insect sting can lead to local reactions like pain, redness, swelling, and even allergic reaction. ${ }^{3}$ During the venom injection, bacteria may enter the tissue and result in an infection and abscess. ${ }^{4}$ Although infections are rare; an abscess may develop.

Prediabetes or intermediate hyperglycemia is an intermediate state of hyperglycemia with glycemic parameters above normal but below the diabetes threshold. The diagnosis of prediabetes can be established based on a fasting plasma glucose of $100-125 \mathrm{mg} / \mathrm{dL}$ and an HbA1c level of 5.76.4\%. ${ }^{5}$ Prediabetic patients have a high risk for developing diabetes. ${ }^{6}$ Prediabetics are more susceptible to developing bacterial infections because of the hyperglycemic condition, which causes changes in the person's immune responsiveness. ${ }^{7}$

Corresponding author: Bagus Soebadi, DDS, MHPED

Department of Oral Medicine

Faculty of Dental Medicine

Universitas Airlangga

Surabaya, Indonesia

Email: bagus-s@fkg.unair.ac.id 
We reported a rare case of a lower lip abscess due to an insect sting with spontaneous drainage in a 39-year-old prediabetic male. The clinical presentation of lip abscess, especially in the vermilion, is a rare condition. The dentist and clinician need to identify this condition and need to manage it appropriately.

\section{CASE REPORT}

A thirty-nine-year-old man complained of swelling on the lower right lip six days before the consult. The swelling started as a vesicle which became bigger. It burst, exuding a yellowish fluid with blood. The swollen area got more pronounced, and the pain was felt, followed by a fever. The patient took Amoxicillin and honey with no relief. This condition suddenly appeared without the precedence of drying, cracking, or itchiness on the lips. The patient's medical history revealed he had experienced facial trauma 9 and 13 years ago. The patient is a non-smoker, consumed no alcoholic beverages, nor is on prohibited drugs.

Extraoral examination showed a swollen area on the vermilion of the right lip, was red, with diffused margins. On palpation, the swollen area was dense and springy. Temperature and pain (Wong-Baker Face Pain Scale was 7) were elevated (Figure 1A). The swelling exceeded the vermilion border of the perioral area (Figure 1B).

On intraoral examination, the lower labial mucosal, vestibular area in regions 43, 44, 45, 46, and gingival mucosa appeared normal (Figure 1C). Based on anamnesis and clinical appearance, the diagnosis was lip abscess. The differential diagnosis was orofacial granulomatosis. The treatment consisted of Clindamycin $300 \mathrm{mg} 2$ times daily, Dexamethasone tablet $0.5 \mathrm{mg} 3$ times daily, and $0.12 \%$ Chlorhexidine di-gluconate applied topically on the swollen area.

The following day after taking the prescribed medications, the swelling disappeared. The swollen lips spontaneously burst to exude a yellowish fluid with blood. The pain subsided to 4 (Wong-Baker Faces Pain Scale). The vermilion on the right lip was covered with a redbrownish crust (Figure 2). No abnormalities were found on intraoral examination.

The complete blood test showed increased eosinophils, lymphocytes, monocytes, erythrocyte sedimentation rate (ESR), and reticulocytes. Neutrophils values decreased. The immunology assay showed a high total immunoglobulin E. The serum glutamic pyruvic transaminase (SGPT) liver function test was elevated. The HbA1c value was $6.2 \%$. The laboratory test results are presented in Table 1.

On further anamnesis, the patient slept on the floor every day. A few days before the swelling of his right lower lip, he felt that his lower lip was bitten by an insect while sleeping. When he woke up the following morning, his lips felt thick. Based on the history and results of the complete blood test, the diagnosis was a lip abscess caused by an insect sting. The treatment was continued, consisting of
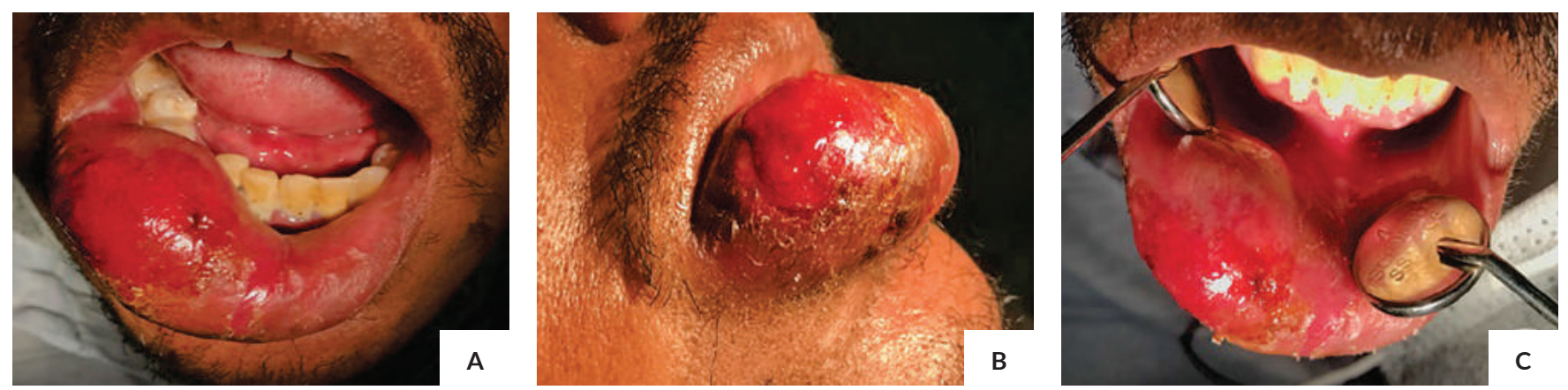

Figure 1. Lip lower abscess from anterior view (A), lateral view (B), the lower labial mucosa (C).
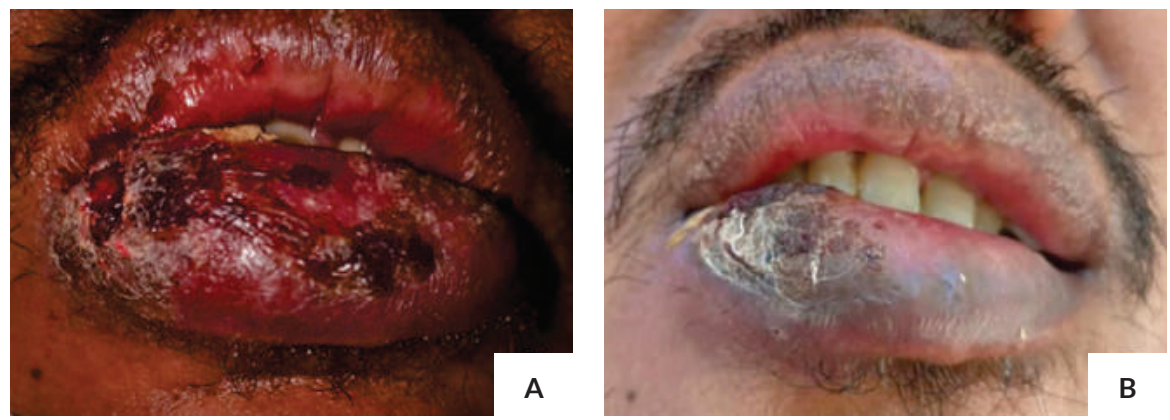

Figure 2. Lip condition after treatment with an antibiotic. The vermilion border is covered with crust. 

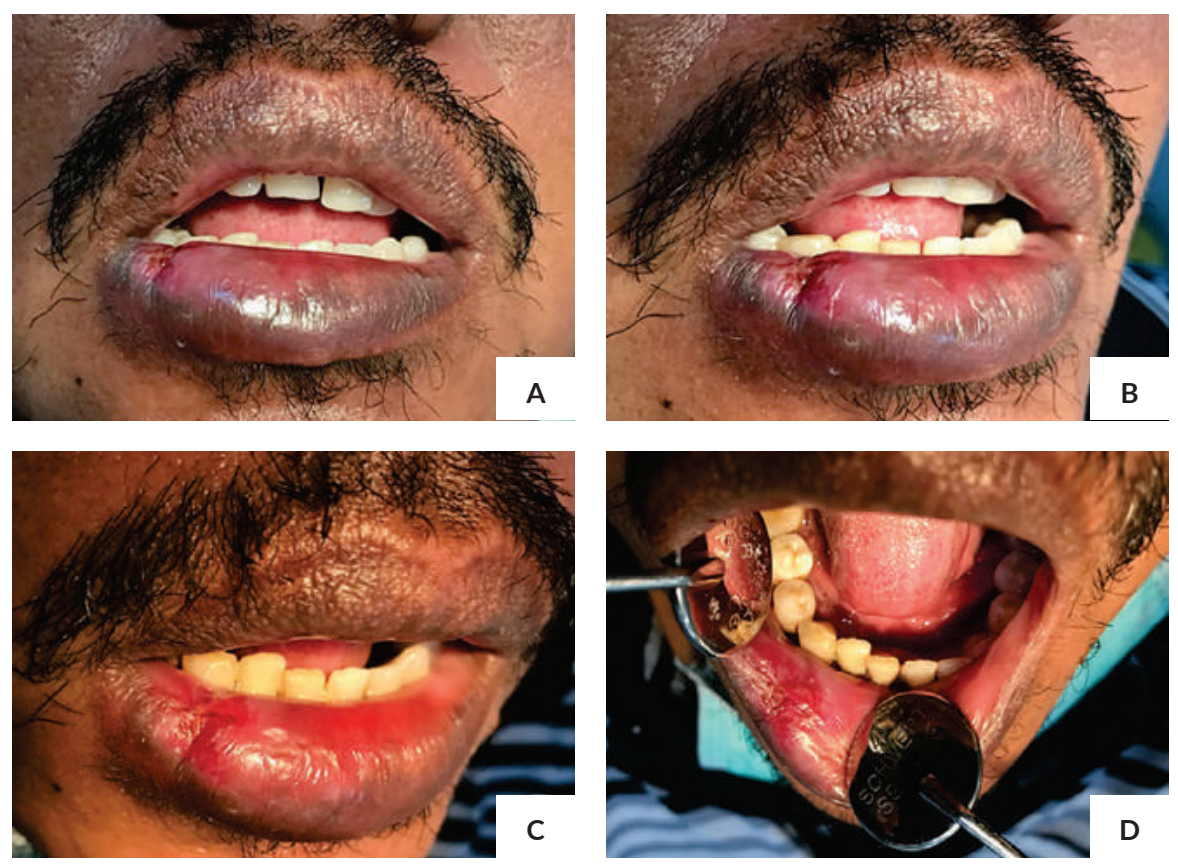

Figure 3. Lower lip dextral showed no abscess (A). the vermilion region showed ulceration after spontaneous drainage (B-C). The labial mucosa is normal (D).

Table 1. The result of complete blood count

\begin{tabular}{lcc} 
No Indicator & Value & Range \\
$\begin{array}{l}\text { Complete blood count } \\
1 \text { Eosinophil }\end{array}$ & $10(\mathrm{H})$ & $2-4 \%$ \\
& $990(\mathrm{H})$ & $80-360 / \mu \mathrm{L}$ \\
2 Neutrophil & $46(\mathrm{~L})$ & $55-65 \%$ \\
3 Lymphocytes & $36(\mathrm{H})$ & $25-35 \%$ \\
4 Monocytes & $8(\mathrm{H})$ & $3-6 \%$ \\
5 ESR & $58(\mathrm{H})$ & $1-30 \mathrm{~mm}$ \\
6 Reticulocytes & $10(\mathrm{H})$ & $0.5-1.5 \%$ \\
\hline Immunology & & \\
7 Total Immunoglobulin E & $663.07(\mathrm{H})$ & $<150 \mathrm{KIU} / \mathrm{L}$ \\
\hline Liver function test & & \\
8 SGOT & 43 & $0-50 \mathrm{u} / \mathrm{L}$ \\
9 SGPT & $150(\mathrm{H})$ & $0-50 \mathrm{u} / \mathrm{L}$ \\
\hline Blood glucose & & \\
10 FBG & 119 & $<126 \mathrm{mg} / \mathrm{dL}$ \\
11 FBG - 2PP & 135 & $<140 \mathrm{mg} / \mathrm{dL}$ \\
12 HbA1C & 6.2 & $<6.5 \%$ \\
\hline
\end{tabular}

ESR - erythrocyte sedimentation rate; SGOT - serum glutamic oxaloacetic transaminase; SGPT - serum glutamic pyruvic transaminase; FBG - fasting blood glucose; FBG-2PP - fasting blood glucose 2 hours postprandial

Clindamycin, Dexamethasone, and 0.12\% Chlorhexidine di-gluconate applied topically.

Six days after the first visit, the swelling disappeared (Figure 3A and 3B). On extraoral examination, the solitary ulcer that was colored red, irregular border, surrounded by erythema macula on the vermilion of the right lip (Figure 3C and 3D) has improved. The pain disappeared (Wong-Baker Faces Pain Scale was 0). The treatment consisted of $0.12 \%$
Chlorhexidine di-gluconate as an oral rinse and a topical ointment containing Hydrocortisone, Lanolin, and Vaseline.

Thirteen days after the first visit, the vermilion of the right lip wholly healed (Figure 4A). Extraoral examination showed the irregular white macula border. The surrounding tissues were normal (Figure 4B). Intraoral examination of the lower labial mucosal, vestibular area in regions $43,44,45$, 46, and the gingival mucosa appeared normal (Figure 4C).

\section{DISCUSSION}

Insect sting can lead to local reactions like erythema and systemic reactions such as anaphylaxis and shock. ${ }^{8}$ The most common manifestations are pain, redness, swelling, even an allergic reaction. ${ }^{3}$ These reactions occur because of the venom. When the toxin enters the tissue, it causes a response to the body. Redness and swelling are common manifestations, generally less than $10 \mathrm{~cm}$ in diameter, and improves markedly within 24 hours. A severe local reaction larger than $10 \mathrm{~cm}$ in diameter persisting for several days may induce mild systemic symptoms. ${ }^{9}$

Cases of insect sting on the lip are sporadic. Gupta et al. reported in 2017 one case of swelling of the lips due to an insect bite. The swelling initially was slight and gradually increased. On palpation, the local temperature was raised with tenderness, and consistency was firm. ${ }^{1}$ The clinical features were similar to our case.

We reported a case of an insect sting in the lower lip that manifested as an abscess. The pathogenesis of lip abscess by insect sting is unclear. Three mechanisms are known to be responsible for the infection. The first mechanism is 

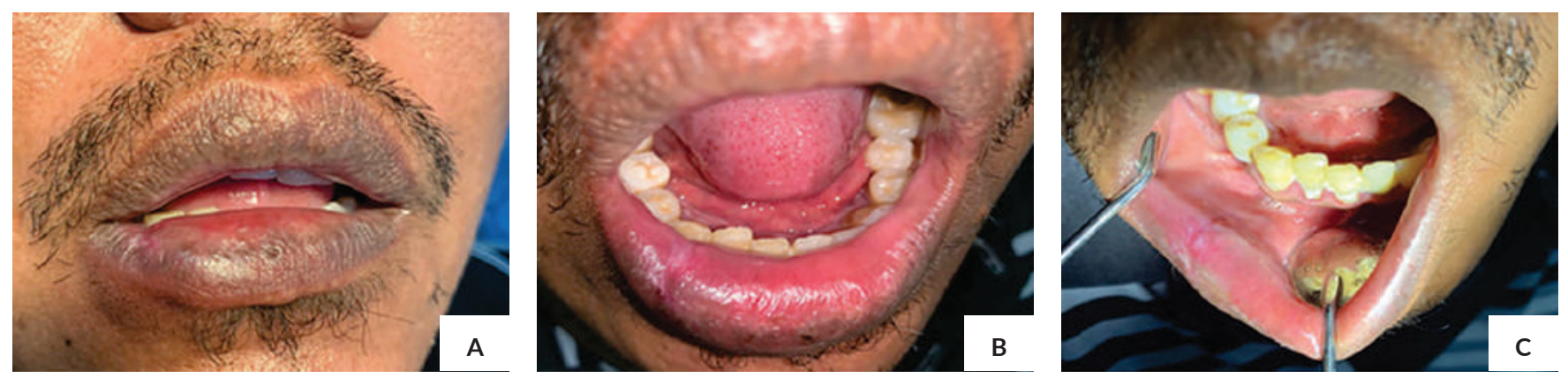

Figure 4. Vermilion of the lip (A-B) and mucosal (C) showed normal after treatment for 13 days.

that the insect may pick up an infectious organism such as a bacteria and passing them along when injecting the venom. ${ }^{4}$ The second is a reaction to the remaining part of the sting as foreign material, resulting in a secondary bacteria infiltration. The third is the tendency of infection due to a damaged epidermis resulting from scratching of the sting site. $^{2}$ There are reports of septicemia occurring after insect stings, caused by pathogens entering the human body via the pores when stingers are left embedded in the skin. ${ }^{4}$ In some research, the culture specimen from insect sting lesion revealed Staphylococcus aureus, Pseudomonas aeruginosa, Enterococcus faecalis, Xanthomonas maltophila, and coagulasenegative Staphylococcus species. ${ }^{10}$ An abscess may develop due to the presence of bacteria after an insect sting. ${ }^{11}$

Even if a culture of bacteria was not done, we confirmed the infection and inflammation condition from the complete blood count - lymphocytes, monocytes, erythrocyte sedimentation rate (ESR), and reticulocytes were elevated. Higher lymphocytes and monocytes indicate a hypersensitivity reaction and viral, fungal, protozoa, and bacterial infection. ${ }^{12} \mathrm{~A}$ high ESR suggests an inflammation. ${ }^{13}$ High reticulocytes indicate malnutrition and inflammation. ${ }^{13,14} \mathrm{~A}$ bacterial infection can be defined based on the neutrophil-lymphocyte ratio (NLR) and monocyte lymphocyte ratio (MLR). Generally, a high neutrophillymphocyte ratio (NLR) and monocyte lymphocyte ratio (MLR) values indicate a more significant probability of a bacterial infection and a low likelihood of a viral infection. ${ }^{15}$ The NLR and MLR are indicators for antibiotic treatment. Our patient's NLR and MLR showed a high ratio. Therefore we suspected bacterial infection.

Another factor compounding the development of the abscess in our patient's lip was a bacterial infection due to his prediabetic condition. Our patient's fasting blood glucose and 2 hours postprandial were a little bit higher than average. His HbA1c also showed 6.2\% ${ }^{16}$ Patients who are prediabetic or intermediate hyperglycaemic, have a higher risk for developing diabetes. ${ }^{6}$ Patients who are prediabetics are more susceptible to developing various oral infections, including fungal and bacterial infections, because of the hyperglycemic condition that causes changes in a person's immune responsiveness. ${ }^{7}$
As mentioned before, there was an allergic reaction before the complication was felt. Allergic reactions from insect stings are immunoglobulin E-mediated hypersensitivity. ${ }^{11}$ These reactions arise because the venom contains specific proteins like Melittin, phospholipase A1, phospholipase A2, hyaluronidase, acid phosphatase, serine protease, and antigen $5 .{ }^{9}$ Phospholipase A can degranulate mast cells and a potent inducer of $\mathrm{IgE}$ antibodies. Other peptides, such as Melittin, in insect venom, may support the development of allergies. This peptide is highly toxic, causing hemolysis, inflammatory reactions, and increased capillary permeability, resulting in membrane disruption and lysis. ${ }^{11}$

Our patient has elevated total immunoglobulin E $(\mathrm{IgE})$ and eosinophils - signaling an allergic reaction. ${ }^{12}$ IgE, mast cells, and eosinophils are essential components in allergic inflammation. With subsequent fixation of $\operatorname{IgE}$ to FceRI receptors on the mast cell, antigen-specific $\operatorname{IgE}$ production is central to the initiation and propagation of immediate hypersensitivity reactions. ${ }^{17}$ Other mechanisms explain how the venom activates mast cells to drive the inflammatory response. This is potentially an ionotropic and calcium-mobilizing stimulus. The venom stimulates the release of histamines in the mast cell by mobilizing the intracellular $\mathrm{Ca}^{2+} .{ }^{18}$

The elevated total $\operatorname{IgE}$ was an indicator that there was an allergic reaction to the insect sting. The diagnosis of an allergic reaction was established from the high total $\mathrm{IgE}$ and the value of a specific IgE. Research by Sturm et al. in 2007 showed there was a correlation between total $\mathrm{IgE}$ with specific $\operatorname{IgE}$, but not to the severity of the sting reaction. ${ }^{19}$ Based on that research, we did not analyze the specific $\operatorname{IgE}$ in our case.

The management of insect sting was directed towards relieving the itchiness, pain, and swelling. Oral and topical corticosteroids such as Dexamethasone or Hydrocortisone were our best choice. The medications reduced the venominduced inflammation, stopped the ciliary spasm, and stabilized the blood-aqueous barrier. ${ }^{20}$ We prescribed oral Dexamethasone and a topical ointment containing hydrocortisone to relieve local inflammation, swelling, and pain. Oral corticosteroids like Dexamethasone is recommended for managing severe local reactions like abscess. ${ }^{20}$ 
The broad-spectrum antibiotics are used prophylactically to prevent an infection. ${ }^{21}$ Clindamycin was given to avoid the spread of the condition and induce spontaneous drainage. Clindamycin was the first choice to treat the skin abscess, in addition to an incision of the swollen area and draining of the pus. In some researches, the administration of Clindamycin improved the condition of the skin abscess compared with no antibiotic. ${ }^{22}$

Other topical treatments like antiseptics, astringents, and cooling agents are sometimes advocated. We prescribed $0.12 \%$ Chlorhexidine di-gluconate as an antiseptic to prevent local infection. ${ }^{20}$ The therapy showed an improvement of the lip's condition, which ultimately healed.

The clinical relevance is that insect stings can cause the vermilion enlargement of the lips. Clinical examination presented as a swollen of the vermilion, red colors, diffused margin, palpation in the dense springy, increased temperature, and pain. A complete history of contact with insects is also crucial in diagnosing lip abscess by insect stings. The significance of this case is the lip abscess able to manage with a combination of antibiotics and oral corticosteroid as the adequate treatment.

\section{CONCLUSION}

Clinical presentation of lip lower abscess due to insect sting is a swollen of the vermilion, red colors, diffused margin, palpation in the dense springy, increase in temperature, and pain. The anamnesis and clinical examination are the critical points for the diagnosis of lip abscess. The insect sting as the etiology of lip abscess must be found from anamnesis. The combination of antibiotics and oral corticosteroid is the adequate treatment of lip abscess by an insect sting. Local therapy like antiseptic and topical corticosteroids can promote the complete healing of lip abscess by preventing infection dissemination and the likelihood of tissue necrosis.

\section{Ethical Consideration}

An ethical review was not required for this case report. The patient in this study approved and signed the patient consent form. The patient has also given consent for the clinical images and clinical information.

\section{Statement of Authorship}

All authors participated in the data collection and analysis and approved the final version submitted.

\section{Author Disclosure}

All authors declared no conflicts of interest.

\section{Funding Source}

This paper was funded by the Universitas Airlanggga.

\section{REFERENCES}

1. Gupta SV, Lohe VK, Bhowate RR, Soni TN. Lip abscess due to insect bite- A case report. J Oral Med Oral Surg. 2017;1(1):27-9.

2. Erkuran MK, Duran A, Ocak T. Ketoacidosis Related to Infections Induced by Bee Sting: A Case Report. Arch Case Reports Clin Med. 2016;2(1):116.

3. da Silva Junior GB, Vasconcelos Junior AG, Rocha AMT, de Vasconcelos VR, de Barros Neto J, Fujishima JS, et al. Acute kidney injury complicating bee stings - A review. Vol. 59, Revista do Instituto de Medicina Tropical de Sao Paulo. 2017. p. 1-7.

4. Gupta PN, Kumar BK, Velappan P, Sudheer MD. Possible complications of bee stings and a review of the cardiac effects of bee stings. BMJ Case Rep. 2016;2016.

5. Brannick B, Wynn A, Dagogo-Jack S. Prediabetes as a toxic environment for the initiation of microvascular and macrovascular complications. Exp Biol Med. 2016;241(12):1323-31.

6. Tabák AG, Herder C, Rathmann W, Brunner EJ, Kivimäki M. Prediabetes: A high-risk state for diabetes development. Lancet. 2012;379(9833):2279-90.

7. Rohani B. Oral manifestation in the patients with diabetes mellitus. World J Diabetes. 2019;10(9):473-89.

8. Rishi E, Rishi P. Intraocular inflammation in a case of bee sting injury. GMS Ophthalmol cases. 2018;8:1-5.

9. Przybilla B, Ruëff F. Insect Stings. Dtsch Arztebl Int. 2012;109(13): 238-48.

10. Truskinovsky AM, Dick JD, Hutchins GM. Fatal Infection after a Bee Sting. Clin Infect Dis. 2001;32(2):e36-8.

11. Alemán Navas RM, Martínez Mendoza MG, Herrera H, de Herrera HP. Floor of the nose mucosa lysis and labial abscess caused by a bee sting. Braz Dent J. 2009;20(3):249-53.

12. Riley LK, Rupert J. Evaluation of Patients with Leukocytosis. Am Fam Physician. 2015;92(11):1004-11.

13. Bray C, Bell LN, Liang H, Haykal R, Kaiksow F, Mazza JJ, et al. Erythrocyte sedimentation rate and $\mathrm{C}$-reactive protein measurements and their relevance in clinical medicine. Wis Med J. 2016;115(6): $317-21$.

14. Cervantes-Ríos E, Ortiz-Muñiz R, Martínez-Hernández AL, Cabrera-Rojo L, Graniel-Guerrero J, Rodríguez-Cruz L. Malnutrition and infection influence the peripheral blood reticulocyte micronuclei frequency in children. Mutat Res - Fundam Mol Mech Mutagen. 2012;731(1-2):68-74.

15. Naess A, Nilssen SS, Mo R, Eide GE, Sjursen H. Role of neutrophil to lymphocyte and monocyte to lymphocyte ratios in the diagnosis of bacterial infection in patients with fever. Infection. 2017;45(3): 299-307.

16. Bansal N. Prediabetes diagnosis and treatment: A review. World J Diabetes. 2015;6(2):296.

17. Stone KD, Prussin C, Metcalfe DD. IgE, Mast Cells, Basophils, and Eosinophils Kelly. J Allergy Clin Immunol. 2010 Feb;125(2 Suppl 2):s73-80.

18. Jansen C, Shimoda LMN, Starkus J, Lange I, Rysavy N, MaaetoftUdsen $\mathrm{K}$, et al. In vitro exposure to Hymenoptera venom and constituents activates discrete ionotropic pathways in mast cells. Channels (Austin). 2019;13(1):264-86.

19. Sturm GJ, Heinemann A, Schuster C, Wiednig M, Groselj-Strele A, Sturm EM, et al. Influence of total IgE levels on the severity of sting reactions in Hymenoptera venom allergy. Allergy. 2007;62(8):884-9.

20. Management of simple insect bites: Where's the evidence? Drug Ther Bull. 2012;50(4):45-8.

21. Ang W-J, MD Kadir S-Z, Fadzillah A-J, Zunaina E. A Case Series of Bee Sting Keratopathy With Different Outcomes in Malaysia. Cureus. 2017;9(2):e1035.

22. Vermandere M, Aertgeerts B, Agoritsas T, Liu C, Burgers J, Merglen A, et al. Antibiotics after incision and drainage for uncomplicated skin abscesses: A clinical practice guideline. BMJ. 2018;360:1-8. 